\title{
Skyrmions around Kerr black holes and spinning BHs with Skyrme hair
}

\author{
C. Herdeiro, ${ }^{a, b}$ I. Perapechka, ${ }^{c}$ E. Radu ${ }^{a}$ and Ya. Shnir ${ }^{d}$ \\ ${ }^{a}$ Departamento de Física da Universidade de Aveiro and CIDMA, \\ Campus de Santiago, 3810-183 Aveiro, Portugal \\ ${ }^{b}$ CENTRA, Departamento de Física, Instituto Superior Técnico - IST, \\ Universidade de Lisboa - UL, Avenida Rovisco Pais 1, 1049 Lisboa, Portugal \\ ${ }^{c}$ Department of Theoretical Physics and Astrophysics, Belarusian State University, \\ Nezavisimosti Avenue 4, Minsk 220004, Belarus \\ ${ }^{d}$ BLTP, JINR, Joliot-Curie 6, Dubna 141980, Moscow Region, Russia \\ E-mail: carlosherdeiro@tecnico.ulisboa.pt, jonahex111@outlook.com, \\ eugen.radu@ua.pt, shnir@theor.jinr.ru
}

AbStract: We study solutions of the Einstein-Skyrme model. Firstly we consider test field Skyrmions on the Kerr background. These configurations - hereafter dubbed Skerrmions - can be in equilibrium with a Kerr black hole $(\mathrm{BH})$ by virtue of a synchronisation condition. We consider two sectors for Skerrmions. In the sector with non-zero baryon charge, Skerrmions are akin to the known Skyrme solutions on the Schwarzschild background. These "topological" configurations reduce to flat spacetime Skyrmions in a vanishing BH mass limit; moreoever, they never become "small" perturbations on the Kerr background: the non-linearities of the Skyrme model are crucial for all such Skerrmions. In the nontopological sector, on the other hand, Skerrmions have no analogue on the Schwarzschild background. Non-topological Skerrmions carry not baryon charge and bifurcate from a subset of Kerr solutions defining an existence line. Therein the appropriate truncation of the Skyrme model yield a linear scalar field theory containing a complex plus a real field, both massive and decoupled, and the Skerrmions reduce to the known stationary scalar clouds around Kerr BHs. Moreover, non-topological Skerrmions trivialise in the vanishing BH mass limit. We then discuss the backreaction of these Skerrmions, that yield rotating BHs with synchronised Skyrme hair, which continously connect to the Kerr solution (self-gravitating Skyrmions) in the non-topological (topological) sector. In particular, the non-topological hairy BHs provide a non-linear realisation, within the Skyrme model, of the synchronous stationary scalar clouds around Kerr.

Keywords: Black Holes, Sigma Models, Solitons Monopoles and Instantons

ArXIV EPRINT: 1808.05388 


\section{Contents}

1 Introduction 1

2 The Skyrme model 3

3 Spherical solutions $\quad 5$

3.1 Minkowski Skyrmions $\quad 6$

$\begin{array}{lll}3.2 & \text { Schwarzschild Skyrmions } & 6\end{array}$

$\begin{array}{lll}\text { 3.2.1 } & \text { Branch structure and the Yang-Mills limit } & 7\end{array}$

$\begin{array}{llr}4 & \text { Topological Skerrmions } & 9\end{array}$

5 Spinning BHs with Skyrme hair and non-topological Skerrmions $\quad 13$

$\begin{array}{ll}5.1 \text { The topological sector } & 14\end{array}$

$\begin{array}{ll}5.2 \text { The non-topological sector } & 15\end{array}$

6 Conclusions 16

\section{Introduction}

The legendary simplicity of black holes (BHs), encapsulated in Wheeler's mantra "BHs have no hair" [1] has been challenged by many different models of hairy BHs - see [2-5] for recent reviews. Historically, the first physically relevant counter example to the no-hair conjecture, in the sense of occuring in a sensible physical model and yielding black holes which are not catastrophically unstable against losing their "hair", was found in the SU(2)Skyrme model. This is a sigma model containing four real scalars subject to a constraint. In flat spacetime its solutions, named Skyrmions, provided also the very first explicit example of solitons in a relativistic non-linear field theory in four spacetime dimensions [6, 7], and have found interesting applications, e.g. as an effective description of low energy Quantum Chromodynamics $[8,9]$ and on the issue of proton decay [10]. These solutions possess a topological baryon charge being therefore topological solitons.

The Skyrme model started to play a role in the context of "hairy BHs" in 1986 when Luckock and Moss [11] (see also [12-16]) observed that a small BH may be superimposed at the center of a Skyrme soliton, yielding a regular, asymptotically flat BH solution, nonsingular on and outside the horizon - see also [13, 17-20]. These solutions are static and spherically symmetric.

As found in [21], however, the flat spacetime Skyrmions possess axially symmetric spinning generalizations, ${ }^{1}$ which may, moreover, gravitate [22] (see also [23]). The existence

\footnotetext{
${ }^{1}$ Spinning Skyrmions were considered already by Skyrme in his pioneering work [7] in order to identify the quantum numbers of nuclei, assuming the rigid body approximation.
} 
of spinning Skyrmions raises the question if four dimensional asymptotically flat spinning BHs with Skyrme hair exist. One of the purposes of this paper is to answer (positively) this question.

In a parallel development motivated by the linear analysis in [24], it has been shown that asymptotically flat rotating BHs (in various dimensions), can be endowed with "hair" of different bosonic fields as along as the field obeys a synchronisation condition, between the angular velocity of the $\mathrm{BH}$ horizon and a phase angular velocity of the matter field. The basic example of this mechanism is provided by Kerr BHs with scalar hair [25, 26]. Further examples of fully non-linear solutions were constructed in [27-33]. In some of these cases, the hairy BHs bifurcate from a reference "bald" solution, such as in [25, 28, 30-33], where the reference solution is Kerr BH. In some other cases the hairy BHs are not continuously connected to the reference bald solution, such as [27, 29], where the reference $\mathrm{BH}$ is the five-dimensional Myers-Perry solution.

The construction of these fully non-linear solutions and, above all, a thorough scanning of their domain of existence is a lengthy and time consuming numerical task. As such, it was suggested in [34] one could probe the existence of these hairy BHs by considering nonlinear $Q$-clouds [35] on the "bald" BH background. In this way, it could be established, for instance that even black objects with topologically non-spherical horizons, such as black rings [36], can be endowed with synchronised scalar hair.

$Q$-clouds are a curved spacetime version of the flat spacetime (spinning) $Q$-ball solutions [37], describing a non-topological soliton in a model with a single, self-interacting, complex scalar field [38, 39]. Amongst the physical differences between the topological spinning Skyrmions and the non-topological spinning $Q$-balls, we would like to point out that for spinning Skyrmions the angular momentum $J$ is a continuous parameter that can be arbitrarily small, whereas for spinning $Q$-balls it is quantised in terms of their Noether charge, possessing a non-zero minimal value. Thus the spinning Skyrmions can rotate slowly and rotating configurations are continuously connected to static ones. This will play a role in the discussion below.

Following the strategy of [34] we shall, in this paper, study the existence of Skyrme hair on Kerr BHs, by firstly studying test field Skyrmions (i.e. non-backreacting) on the Kerr background. These solutions, hereafter dubbed Skerrmions, exist under the synchronisation condition discussed above. We shall consider Skerrmions that fall into two distinct sectors, according to the existence of a non-vanishing or vanishing baryon number.

In the topological sector, Skerrmions reduce to the usual flat spacetime Skyrmions when gravity is switched off. Such Skerrmions never become "small", in the sense of becoming solutions of a linear field theory on the Kerr spacetime. This contrasts to the behaviour of $Q$-clouds, that become linear solutions on an existence line of Kerr BHs, wherein the scalar field becomes small, the non-linearities become irrelevant and the $Q$-clouds reduce to the stationary scalar clouds found in [24] (see also, e.g. [40-46]). Such existence line defines the subset of Kerr BHs from which the fully non-linear "hairy" BH solutions bifurcate. The absence of an existence line anticipates that spinning BHs with topological Skyrme hair do not bifurcate from Kerr BHs, similarly to the aformentioned examples [27, 29]. These Skerrmions also possess a non-zero baryon number, which in the limit of vanishing event horizon, becomes an integer - the topological charge. 
In the non-topological sector, Skerrmions trivialise in the limit when gravity is switched off. Such Skerrmions could only be found as linear solutions on an existence line of Kerr BHs, wherein the Skyrme model reduces to a linear field theory composed of a complex together with a real scalar fields, with these fields decoupled and massive, and carrying no baryon charge. Then, these non-topological Skerrmions, like $Q$-clouds, match the stationary clouds in [24], at the existence line. Moreover, the spinning BHs with non-topological Skyrme hair bifurcate from Kerr, similarly to the examples in [25, 28, 30-33].

We also construct and discuss some basic properties of backreacting Skerrmions, yielding spinning BHs with Skyrme hair, both in the topological and non-topological sector, in particular verifying the results anticipated by the test field analysis.

This paper is organised as follows. In section 2 we present the basic formalism of the Skyrme model, including the definition of the topological charge, and some comments on the $O(3)$ truncation that yields the non-topological sector. As a preparation for the presentation of Skerrmions, in section 3 we review the spherically symmetric non-backreacting Skyrme solutions on Minkowski as well as those on a Schwarzschild BH, wherein only the topological sector exists. The latter solutions have a branch structure for their global quantities in terms of a dimensionless coupling. In one of the limits of zero coupling the flat spacetime solutions are recovered. In another limit, Yang-Mills solitons are obtained. In section 4 we describe the topological Skerrmion solutions and in 5 we discuss the nontopological sector and backreacting Skerrmions that yield spinning BHs with Skyrme hair. Concluding remarks are presented in section 6 .

\section{The Skyrme model}

Working in four spacetime dimensions with a metric tensor $g_{\mu \nu}$, we consider the $\mathrm{SU}(2)$ Skyrme Lagrangian,

$$
\mathcal{L}_{S}=\operatorname{Tr}\left\{\frac{\kappa^{2}}{4} \mathbf{K}_{\mu} \mathbf{K}^{\mu}+\frac{1}{32 e^{2}}\left[\mathbf{K}_{\mu}, \mathbf{K}_{\nu}\right]\left[\mathbf{K}^{\mu}, \mathbf{K}^{\nu}\right]\right\}+\frac{m_{\pi}^{2}}{2} \operatorname{Tr}\left\{\frac{\mathbf{U}+\mathbf{U}^{\dagger}}{2}-\mathbf{1}\right\},
$$

where $\kappa, e$ and $m_{\pi}$ are input parameters, ${ }^{2} m_{\pi}$ being interpreted as the pion mass. $\mathbf{K}_{\mu}$ is the $\mathrm{SU}(2)$-valued left-invariant current,

$$
\mathbf{K}_{\mu}=\partial_{\mu} \mathbf{U U}^{-1}
$$

associated with the Skyrme field,

$$
\mathbf{U}=\sigma \mathbf{1}+i \pi_{a} \tau^{a} \underset{r \rightarrow \infty}{\longrightarrow} \mathbf{1}
$$

where $\tau^{a}$ are the Pauli matrices and $\left\{\sigma, \pi_{a}\right\}$ spacetime fields that behave as $\sigma \rightarrow 1, \pi_{a} \rightarrow 0$ in the spatial asymptotic limit $r \rightarrow \infty$.

The Lagrangian (2.1) contains three terms. The first term is the usual non-linear sigma model term; the second is known as 'the Skyrme term', being required by Derrick-type

\footnotetext{
${ }^{2}$ In nuclear physics applications, the value of these parameters is fixed by comparison with experimental data. For the conventions here, the dimensions of these constants are: $[\kappa]=1 / L,[e]=L^{0}$ and $\left[m_{\pi}\right]=1 / L^{2}$.
} 
scaling arguments [47] for the existence of finite mass solutions; the third is a mass term which is mandatory for rotating solutions, whereas it can be set to zero in the static case. A generalization of the Skyrme model, which includes an additional sextic in derivatives term, was suggested recently to construct weakly bounded multisoliton configurations [48-50].

The Skyrme field satisfies the equations

$$
\nabla_{\mu}\left(\kappa^{2} \mathbf{K}^{\mu}+\frac{1}{4 e^{2}}\left[\mathbf{K}_{\nu},\left[\mathbf{K}^{\mu}, \mathbf{K}^{\nu}\right]\right]\right)=\frac{m_{\pi}^{2}}{2}\left(\mathbf{U}-\mathbf{U}^{\dagger}\right)
$$

Moreover, the fields $\left\{\sigma, \pi_{a}\right\}$ which enter the decomposition of the Skyrme field, (2.3), are subject to the sigma-model condition,

$$
\sigma^{2}+\pi_{a} \pi_{a}=1
$$

Thus, in the absence of an event horizon, $\left\{\sigma, \pi_{a}\right\}$ map the compactified coordinate space $S^{3}$ to the $\mathrm{SU}(2)$ group space, which is isomorphic to the 3 -sphere, $S^{3}$. The homotopy group of this map is $\pi_{3}\left(S^{3}\right)=\mathbb{Z}$; consequently, each $S^{3} \longrightarrow S^{3}$ map falls into an homotopy class indexed by an integer, which is identified with the topological charge $B$ :

$$
B \equiv \int_{\Sigma} B^{t} d^{3} x
$$

with the topological current $B^{\mu}$

$$
B^{\mu} \equiv \frac{1}{\sqrt{-g}} \frac{1}{24 \pi^{2}} \epsilon^{\mu \nu \alpha \beta} \operatorname{Tr}\left(\mathbf{K}_{\nu} \mathbf{K}_{\alpha} \mathbf{K}_{\beta}\right) .
$$

The (static) solution found by Skyrme using numerical methods [6, 7] and whose existence proof was given in [51], has $B=1$. We emphasize that the topological charge $B$ does not arise from an invariance of the matter Lagrangian under any symmetry transformation and is therefore not a Noether charge [52], unlike the charge of $Q$-balls. Its origin comes instead from the non-trivial topology of the Skyrmions.

In the case of the BHs with Skyrme hair, strictly speaking, the topology (i.e. the mapping between physical and internal spaces) is lost. Still, one defines a baryon charge $B$ by performing the integration (2.6) in the exterior region. The numerical results in $[13,17-$ 20 ] show that the BH horizon "absorbs" a part of the baryon charge, but $B$ never vanishes for a static horizon and thus the vacuum $\mathrm{BH}$ is never approached. As we shall see below, the same holds for the topological sector of Skerrmions.

In the non-topological sector, on the other hand, corresponding to a particular $O(3)$ truncation of the full Skyrme model, Skerrmions always have $B=0$. In this case, moreover, Skerrmions become infinitesimally small. To better understand this point, let us consider small perturbations around the Skyrme vacuum state (thus $B=0$, trivially ${ }^{3}$ ); these perturbations are described by parameterising the $\left\{\sigma, \pi_{a}\right\}$ fields as

$$
\pi_{a}=\epsilon \Pi_{a}, \quad \sigma=1+\epsilon^{2} \Sigma,
$$

\footnotetext{
${ }^{3}$ The Skyrme model possesses solutions with $B=0$ and nonzero mass, describing Skyrme-anti-Skyrme composite states $[53,54]$ (likely unstable). These solutions can be studied within the same Ansatz employed in this work. They satisfy, however, a different set of boundary conditions and they shall not be considered in this work.
} 
where $\epsilon \ll 1$ and $\Sigma=-\Pi_{a} \Pi_{a} / 2$ from the sigma-model condition (2.5). Then, defining the complex scalar field $\psi$ and a real one $\chi$ as

$$
\psi=\Pi_{1}+i \Pi_{2}, \quad \chi=\Pi_{3},
$$

to leading order, the Skyrme Lagrangian (2.1) reduces to $\mathcal{L}_{S} \simeq \mathcal{L}_{\psi}+\mathcal{L}_{\chi}$, where

$$
\mathcal{L}_{\psi}=-\partial_{\mu} \psi^{*} \partial^{\mu} \psi-m_{\pi}^{2} \psi^{*} \psi, \quad \mathcal{L}_{\chi}=-\partial_{\mu} \chi \partial^{\mu} \chi-m_{\pi}^{2} \chi^{2} .
$$

On a static $\mathrm{BH}$, both $\psi$ and $\chi$ vanish identically. However, the presence of a rotating horizon allows for nontrivial fields $\psi, \chi$ forming bound states for a particular set of Kerr backgrounds. These correspond precisely to the existence line discussed in [24, 25]. Thus, at the linear level, stationary scalar clouds of a complex massive scalar field are solutions of the Skyrme model on a Kerr backround. At the non-linear fully backreacting model, the Skyrme model, in the non-topological sector, will provides a more fundamental context wherein hairy BHs supported by the synchronisation condition arise, akin to Kerr BHs with scalar hair [25]. We remark that for these nonlinear realizations of the complex field $\psi$, the real scalar field $\chi$ (which is infinitesimally small on the Kerr existence line) must vanishes identically to all orders. Otherwise, the energy-momentum tensor of the real scalar field $\chi$ would possess a dependence on both time and azimuthal coordinates and its backreacting version would not be compatible with a stationary, axi-symmetric geometry.

\section{Spherical solutions}

As a preparation for Skerrmions let us start by reviewing spherically symmetric solutions. Besides being much simpler, they possess already a number of basic properties of the corresponding rotating generalizations in the topological sector.

Working in spherical coordinates, we consider a general metric Ansatz:

$$
d s^{2}=-f_{0}(r) d t^{2}+f_{1}(r)\left[d r^{2}+r^{2}\left(d \theta^{2}+\sin ^{2} d \varphi^{2}\right)\right] .
$$

For the Skyrme field, we take the usual spherically symmetric Skyrme ansatz (2.3): ${ }^{4}$

$$
\sigma=\cos F(r), \quad \pi_{a}=n_{a} \sin F(r)
$$

where $n_{a}$ are the components of the unit vector:

$$
n_{a}=(\sin \theta \cos m \varphi, \sin \theta \sin m \varphi, \cos \theta)
$$

and $m$ is a positive integer, $m \in \mathbb{N}$. Thus, in the probe limit, the problem reduces to solving a single ordinary differential equation for the Skyrme function $F(r)$. In what follows we shall restrict our study to $m=1$ solutions.

\footnotetext{
${ }^{4}$ Strictly speaking the Skyrme field (3.2) is not spherically symmetric. That is, the effect of a spatial rotation of the Skyrme field can be compensated by an isospin transformation, rather than the Skyrme field being only dependent on the radial coordinate. However, both the energy density and baryon charge density are spherically symmetric, the Ansatz (3.2) being compatible with the line element (3.1). Thus, one may regard these solutions as another case of symmetry non-inheritance [55].
} 


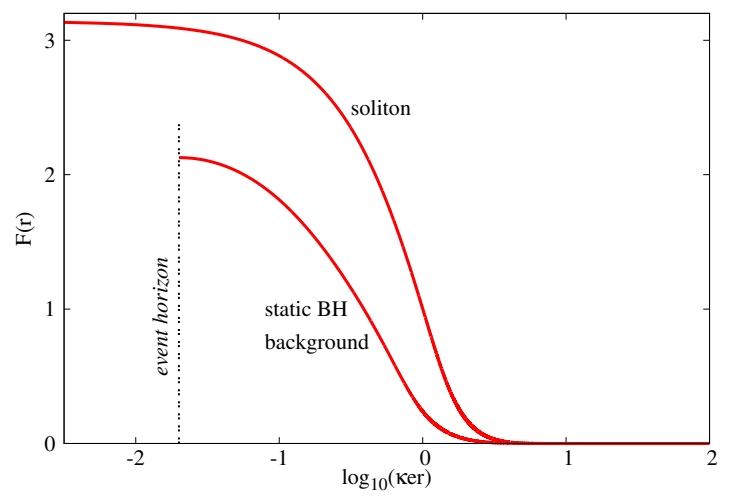

Figure 1. The profile of the Skyrme function, $F(r)$, for the fundamental Minkowski and Schwarzschild Skyrmions. The mass term vanishes in both cases, $m_{\pi}=0$, while $r_{H} \kappa e=0.02$.

For both Minkowski and Schwarzschild Skyrmions, the total mass of the solutions in the probe limit, $M$, is computed as the integral of the $T_{t}^{t}$ component of the energy momentum tensor (as given by (5.3) below), while the topological charge, (2.6), for the Skyrme Ansatz (3.2), reduces to

$$
B=\left.\frac{1}{\pi}\left\{-F(r)+\frac{1}{2} \sin [2 F(r)]\right\}\right|_{r_{0}} ^{\infty} .
$$

For Minkowski Skyrmions, $r_{0}=0$; for solutions on Schwarzschild BH background $r_{0}=r_{H}$.

\subsection{Minkowski Skyrmions}

For a Minkowski background, $f_{0}(r)=f_{1}(r)=1$ in (3.1). Then, the near origin solution for $F(r)$ is:

$$
F(r)=\pi-b r^{2}+\mathcal{O}\left(r^{4}\right),
$$

while the far field expression reads

$$
F(r)=\frac{e^{-m_{\pi} r}}{r}+\ldots
$$

Thus, from (3.4), the topological charge of the horizonless spherical Skyrmion with $m=1$ is $B=1$.

The profile of the (fundamental, flat space) Skyrme soliton is shown in figure 1. One can see that $F(r)$ interpolates smoothly between $\pi$ and 0 , without nodes. Here we use the same scaling and units as in the case of a Schwarzschild BH background (as discussed below).

\subsection{Schwarzschild Skyrmions}

Considering isotropic coordinates, the Schwarzschild $\mathrm{BH}$ can be written in the form (3.1) with

$$
f_{0}(r)=\frac{\left(1-\frac{r_{H}}{r}\right)^{2}}{\left(1+\frac{r_{H}}{r}\right)^{2}}, \quad f_{1}(r)=\left(1+\frac{r_{H}}{r}\right)^{4}
$$


with the $\mathrm{BH}$ mass, $M_{\mathrm{Sch}}$, being related to the event horizon radius in this coordinates, $r_{H}$, as

$$
M_{\mathrm{Sch}}=2 r_{H} .
$$

In this case there is a new length scale, $r_{H}$.

The Skyrme field Ansatz is still given by (3.2). Then we obtain the effective Lagrangian:

$$
\mathcal{L}_{\text {eff }}=r^{2} \sqrt{f_{0} f_{1}^{3}}\left[\frac{\kappa^{2}}{2 f_{1}}\left(F^{\prime 2}+\frac{2 \sin ^{2} F}{r^{2}}\right)+\frac{1}{e^{2}} \frac{2 \sin ^{2} F}{r^{2} f_{1}^{2}}\left(F^{\prime 2}+\frac{2 \sin ^{2} F}{2 r^{2}}\right)+m_{\pi}^{2}(1-\cos F)\right] .
$$

While the far field expression is still given by (3.6), the near horizon form of the solution is

$$
F(r)=F_{H}+F_{2}\left(r-r_{H}\right)^{2}+\ldots, \text { with } F_{2}=\frac{\sin F_{H} \cos F_{H}}{4 r_{H}^{2}}+\frac{\left(16 m_{\pi}^{2} r_{H}^{2}+\kappa^{2} \cos F_{H}\right) \sin F_{H}}{4 \kappa^{2} r_{H}^{2}+\sin ^{2} F_{H} / e^{2}}
$$

The corresponding value of the baryon charge is

$$
B=\frac{1}{\pi}\left(F_{H}-\sin F_{H} \cos F_{H}\right)
$$

In figure 1 we also show the profile of a typical Skyrmion on a Schwarzschild BH background. The Skyrme function $F(r)$ is still monotonic, but $F\left(r_{H}\right) \neq \pi$. Observe that $B \neq 0$ and thus these Schwarzschild Skyrmions belong to the topological sector (there is no non-trivial non-topological sector on Schwarzschild).

\subsubsection{Branch structure and the Yang-Mills limit}

To understand how the mass and baryon charge of Skyrmions on Schwarzschild vary in the space of solutions, let us remark on scaling and units. The Skyrme model has a natural length scale in terms of the input constants of the model, $L_{S} \equiv 1 / \kappa e$. Then, we define a dimensionless radial coordinate, $x$, as $x \equiv r / L_{S}=\kappa e r$. For the BH backgrounds, we define the dimensionless "coupling constant"

$$
\alpha^{2} \equiv \frac{M_{\text {Schw }}}{L_{S}}=2 r_{H} \kappa e .
$$

One defines also a dimensionless pion mass $\mu \equiv m_{\pi} /\left(\kappa^{2} e\right)$, while the relation between the (displayed) numerical value for the total mass-energy for Skyrmions, $M^{(\mathrm{num})}$, and the dimensionful one $M$, is

$$
M^{(\mathrm{num})}=M \frac{\kappa}{e} .
$$

The variation of the Skyrmions mass $M$ and baryon charge $B$ with the coupling constant $\alpha$ is shown in figure 2 for illustrative values of the pion mass. Given a set of input parameters in the Skyrme Lagrangian, BHs able to support Skyrmions cannot be arbitrarily large, with the existence of a maximal value for the $\mathrm{BH}$ mass. Moreover, for any allowed value of $\alpha$, one notices the existence of two branches of solutions which merge for the maximal $\alpha$. The baryon charge $B$ decreases along both branches, approaching the minimal value for the limiting solution on the second branch. 

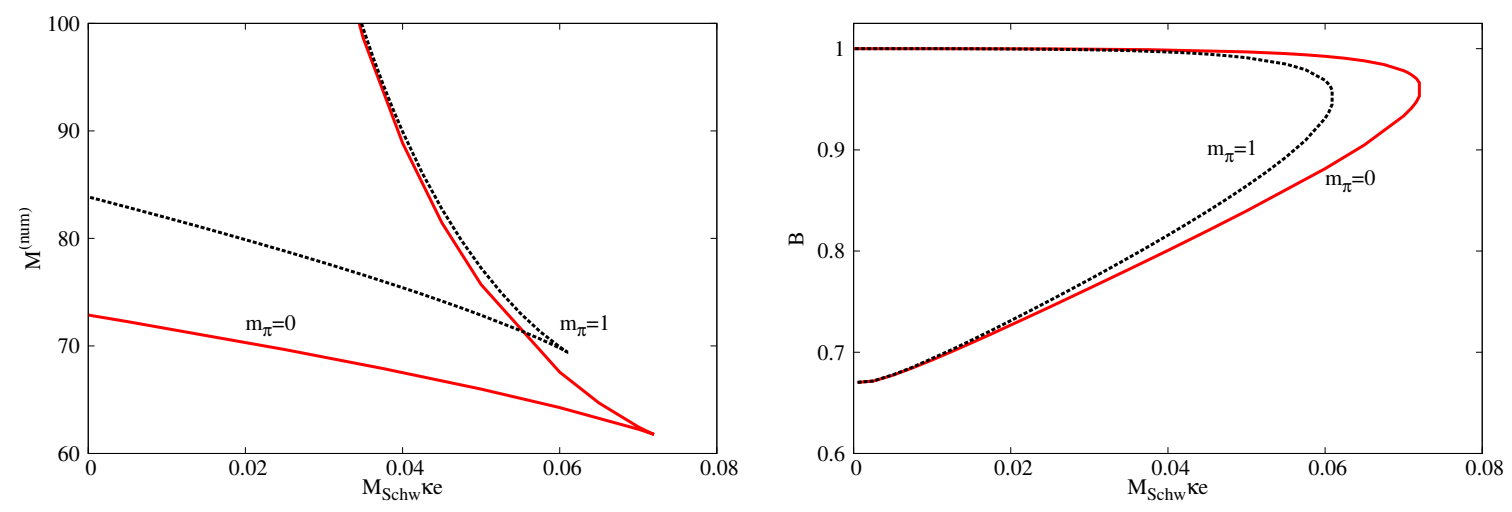

Figure 2. Mass (left panel) and baryon charge (right panel) for Skyrmions on a Schwarzschild BH, in terms of the coupling $\alpha=M_{\mathrm{Schw}} \kappa e$, for two illustrative values of the pion mass.

Due to the branch structure, there are two ways to approach the limit $\alpha \rightarrow 0$. Firstly, it can be approached as $r_{H} \rightarrow 0$ (i.e. a flat spacetime background). These are the solutions which form the fundamental branch (since they can be smoothly connected to the flat space Skyrmions). They are of special interest, being stable. Secondly, it can be approached along a branch of excited configurations which emerge from the fundamental branch, at the maximal value of $\alpha$. In this case, the limit $\alpha \rightarrow 0$ is approached as $\kappa \rightarrow 0$, corresponding to the absence of the quadratic term in the Skyrme Lagrangian. The Skyrmion mass $M^{\text {(num) }}$ diverges in this limit, but the product $\alpha M^{(\text {num })}$ approaches a finite value independent of $\mu .^{5}$

In order to understand this behaviour we introduce a new dimensionless radial coordinate $y$, with

$$
\bar{r}=r_{H} \kappa y \quad \text { i.e. } r=r_{H} e y,
$$

the length scale being fixed here by the horizon radius $r_{H}$. Then, after taking the limit $\kappa \rightarrow 0$ (with fixed $\mu$ ) one finds that the system is described by the reduced effective Lagrangian

$$
\mathcal{L}_{\text {eff }}=4 \sqrt{\frac{f_{0}}{f_{1}}}\left[\left(\frac{d w_{0}}{d y}\right)^{2}+\frac{\left(1-w_{0}^{2}\right)^{2}}{2 y^{2}}\right],
$$

with $w_{0}(y)=\cos F(y)$. One recognizes (3.15) as corresponding to the SU(2)-Yang-Mills (YM) effective Lagrangian. Here one considers the usual YM spherical connection with $w_{0}$ corresponding to the magnetic gauge potential (and a vanishing electric potential). Then the limiting solution corresponds to a spherically symmetric YM configuration on a fixed Schwarzschild background. The (fundamental) exact solution has been found long time ago $[56],{ }^{6}$ its expression in isotropic coordinates being

$$
w_{0}(r)=-\frac{\sqrt{3}+\frac{1-\sqrt{3}}{4 r r_{H}}\left(r+r_{H}\right)^{2}}{3-\frac{1-\sqrt{3}}{4 r r_{H}}\left(r+r_{H}\right)^{2}} .
$$

\footnotetext{
${ }^{5}$ An interesting different branch structure is observed in the modified twelfth-order Einstein-Skyrme model [57].

${ }^{6}$ Historically, this largely unknown paper, describes the first example of hair on Schwarzschild BH (in the probe limit).
} 
From (3.4), this implies a value of the baryon charge $B \simeq 0.668518$ which provides a minimum for all solutions. The total mass in this limit (after multiplying with $\kappa$ ) is

$$
M \simeq 16 \pi M_{\text {Schw }} 0.9587 .
$$

These results are independent of the presence of a mass term in the Skyrme Lagrangian.

\section{Topological Skerrmions}

We now turn our attention to the solutions of the Skyrme model on a fixed Kerr BH geometry with non-trivial baryon charge (topological sector). In quasi-isotropic coordinates, the Kerr line element is

$$
d s^{2}=-F_{0}(r, \theta) d t^{2}+F_{1}(r, \theta)\left(d r^{2}+r^{2} d \theta^{2}\right)+F_{2}(r, \theta) r^{2} \sin ^{2} \theta[d \varphi-W(r, \theta) d t]^{2},
$$

where [65]

$$
\begin{aligned}
& F_{1}(r, \theta)=\frac{2 M_{\mathrm{Kerr}}^{2}}{r^{2}}+\left(1-\frac{r_{H}^{2}}{r^{2}}\right)^{2}+\frac{2 M_{\mathrm{Kerr}}}{r}\left(1+\frac{r_{H}^{2}}{r^{2}}\right)-\frac{M_{\mathrm{Kerr}}^{2}-4 r_{H}^{2}}{r^{2}} \sin ^{2} \theta, \\
& F_{2}(r, \theta)=\frac{S(r, \theta)}{F_{1}(r, \theta)}, \\
& F_{0}(r, \theta)=\left(1-\frac{r_{H}^{2}}{r^{2}}\right)^{2} \frac{F_{1}(r, \theta)}{S(r, \theta)}, \\
& W(r, \theta)=\frac{2 M_{\text {Kerr }} \sqrt{M_{\mathrm{Kerr}}^{2}-4 r_{H}^{2}}}{r^{3}} \frac{1+\frac{M_{\mathrm{Kerr}}}{r}+\frac{r_{H}^{2}}{r^{2}}}{S(r, \theta)}
\end{aligned}
$$

with

$$
S(r, \theta)=\left[\frac{2 M_{\mathrm{Kerr}}^{2}}{r^{2}}+\left(1-\frac{r_{H}^{2}}{r^{2}}\right)^{2}+\frac{2 M_{\mathrm{Kerr}}}{r}\left(1+\frac{r_{H}^{2}}{r^{2}}\right)\right]^{2}-\left(1-\frac{r_{H}^{2}}{r^{2}}\right)^{2} \frac{M_{\mathrm{Kerr}}^{2}-4 r_{H}^{2}}{r^{2}} \sin ^{2} \theta,
$$

and contains two input parameters, the event horizon radius $r_{H}$ and the $\mathrm{BH}$ mass $M_{\mathrm{Kerr}}$. The event horizon angular velocity is determined by these parameters:

$$
\Omega_{H}=\frac{\sqrt{M_{\mathrm{Kerr}}^{2}-4 r_{H}^{2}}}{2 M_{\mathrm{Kerr}}\left(M_{\mathrm{Kerr}}+2 r_{H}\right)} .
$$

The fields $\left\{\sigma, \pi_{a}\right\}$ in the Skyrme field Ansatz (2.3), are defined by three real functions $\phi_{a}$, subject to a constaint $[21,22]$,

$$
\pi_{1}+i \pi_{2}=\phi_{1}(r, \theta) e^{i(m \varphi-w t)}, \quad \pi_{3}=\phi_{2}(r, \theta), \quad \sigma=\phi_{3}(r, \theta), \text { with } \sum_{a=1}^{3} \phi_{a} \phi_{a}=1,
$$

where $w$ is the harmonic frequency of the Skyrme field. The spherical limit is recovered for $\phi_{a}=n_{a} F(r)$. 
The solutions are found again by solving a boundary value problem. The boundary conditions imposed at spatial infinity are $\left.\phi_{1}\right|_{r=\infty}=\left.\phi_{2}\right|_{r=\infty}=0,\left.\quad \phi_{3}\right|_{r=\infty}=1$, while on the symmetry axis we impose $\left.{ }^{7} \phi_{1}\right|_{\theta=0, \pi}=\left.\partial_{\theta} \phi_{2}\right|_{\theta=0, \pi} ^{\infty}=\left.\partial_{\theta} \phi_{3}\right|_{\theta=0, \pi}=0$. Of special interest is the behaviour as $r \rightarrow r_{H}$. Assuming the existence of a power series expansion in $r-r_{H}$, first one finds the following synchronisation condition between the field frequency and the event horizon angular velocity (4.3).

$$
w=m \Omega_{H} .
$$

This condition implies that there is no net flux of the Skyrme field across the horizon and also that the components $T_{t}^{t}$ and $T_{\varphi}^{t}$ of the energy-momentum tensor are finite at the horizon. Second, the same approximate solution in $r-r_{H}$ implies the following horizon boundary conditions: $\left.\partial_{r} \phi_{1}\right|_{r=r_{H}}=\left.\partial_{r} \phi_{2}\right|_{r=r_{H}}=\left.\partial_{r} \phi_{3}\right|_{r=r_{H}}=0$, which are imposed in the numerical procedure. Also, all solutions in this work possess a reflection symmetry along the equatorial plane $(\theta=\pi / 2)$, which implies $\left.{ }^{8} \partial_{\theta} \phi_{1}\right|_{\theta=\pi / 2}=\left.\phi_{2}\right|_{\theta=\pi / 2}=\left.\partial_{\theta} \phi_{3}\right|_{\theta=\pi / 2}=0$.

For the Ansatz (4.4), the baryon charge is

$$
B=\frac{m}{\pi} \int_{r_{H}}^{\infty} d r \int_{0}^{\pi} d \theta \rho(r, \theta), \text { with } \rho(r, \theta)=\partial_{r} \phi_{2} \partial_{\theta} \phi_{3}-\partial_{r} \phi_{3} \partial_{\theta} \phi_{2} .
$$

Skerrmion solutions are found by using the same scaling and units as in the static limit, together with

$$
w \rightarrow w \kappa e, \quad \Omega_{H} \rightarrow \Omega_{H} \kappa e .
$$

Most of the non-spherical solutions ${ }^{9}$ reported in this work were found by using a selfimplemented finite difference method based on Newton-Raphson method with PARDISO as a linear solver [58]. In numerics, a compactified radial coordinate $x$ was introduced with $x=\left(r-r_{H}\right) /(1+r)$. Most of the results have been obtained for an equidistant grid in $(x, \theta)$, with $100 \times 50$ points. The sigma-model constraint $\sum_{a=1}^{3} \phi_{a} \phi_{a}=1$ is imposed by using the Lagrange multiplier method, as explained e.g. in [61, 62].

The profile of a typical solution is shown in figure 3, using a choice of the (scaled) pion mass $\mu=1$ and winding number $m=1$. One can see that e.g. the deformation from sphericity of the energy density is rather small, while the angular momentum density is localized in a torus around the equatorial plane. The properties of these topological Skerrmions are summarised in the following.

Firstly, we have observed that any (spinning) solitonic solution appears to possess generalizations with a small horizon at its center, provided the Skyrme field and the horizon are in synchronous rotation. The field frequency (i.e. the event horizon angular velocity)

\footnotetext{
${ }^{7}$ These boundary conditions result from an approximate construction of the solution on the symmetry axis as a power series in $\theta$ (or $\pi-\theta$ ) and are compatible with the finiteness of $T_{t}^{t}$ and $T_{\varphi}^{t}$-components of the energy-momentum tensor.

${ }^{8}$ The imposed conditions on the boundaries of the domain of integration are compatible with those imposed both in the static limit (see (3.1) and (3.6), (3.10)) and in the spinning, solitonic limit of the solutions (as given e.g. in [22]).

${ }^{9}$ Some of the solutions were also constructed by using a professional package [59,60], which also uses the Newton-Raphson method. We have found a very good agreement between the results obtained within these different approaches.
} 

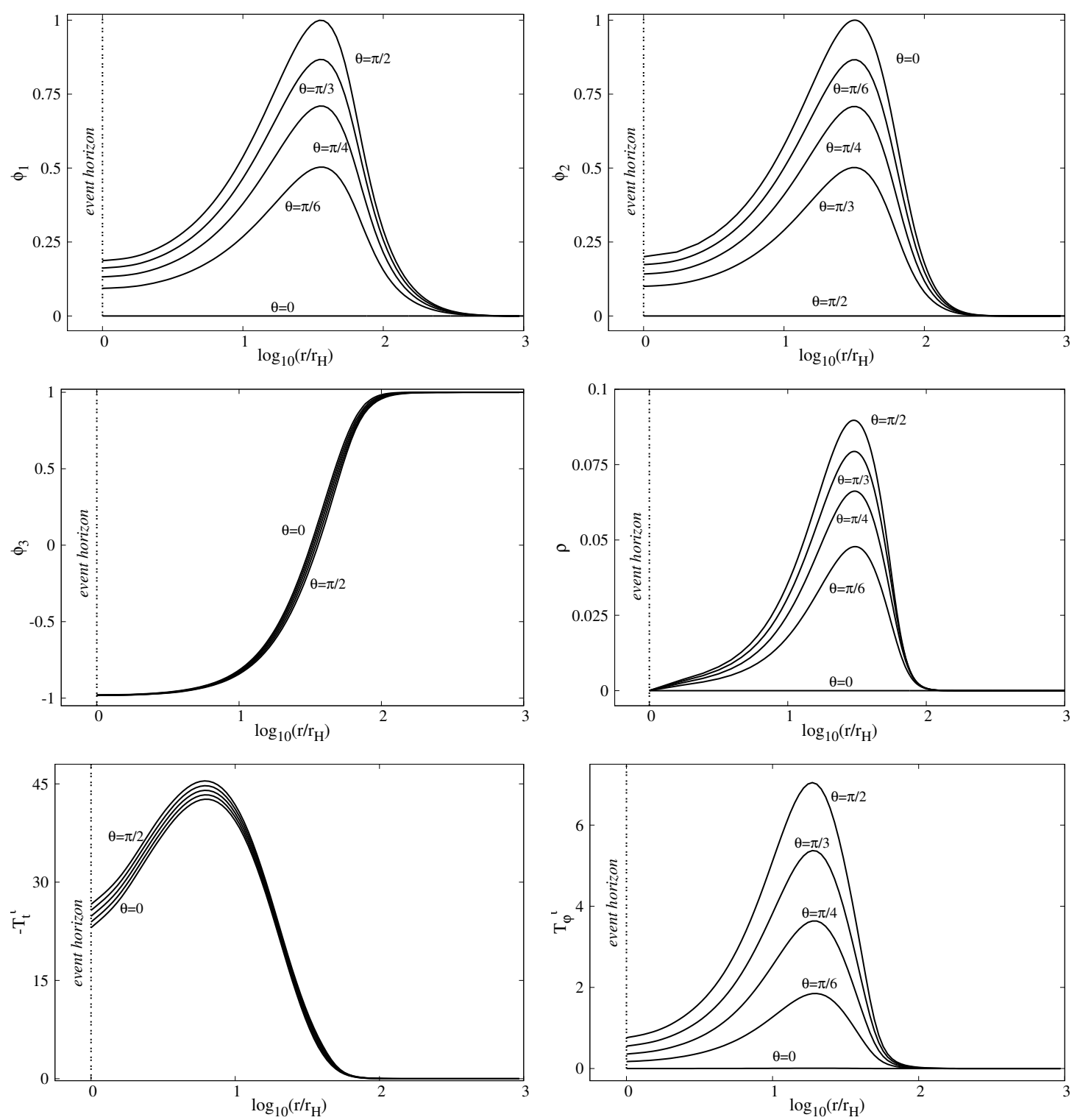

Figure 3. The functions $\phi_{a}$ and the baryon charge density $\rho$, energy and angular momentum densities are shown for Skerrmions on a Kerr background with $M_{\text {Kerr }}=0.04$ and $\Omega_{H}=0.95$.

cannot be arbitrary large. A linearization in the far-field of the field equation for $\psi=$ $\pi_{1}+i \pi_{2}$ yields

$$
\left(\nabla_{0}^{2}+w^{2}-\mu^{2}\right) \psi=0
$$

where $\nabla_{0}^{2}$ is the Laplace operator on Euclidean 3 -space $\mathbb{R}_{3}$, which imposes the boundstate condition $w \leqslant \mu$. Therefore, similarly to other examples of spinning solutions with scalar fields [62], the presence of a mass term in the action is a necessary condition for the existence of localized configurations.

Secondly, topological Skerrmions are found only for a limited set of Kerr backgrounds - see figure 4, with a maximal BH size defining the critical backgrounds, similarly to what 


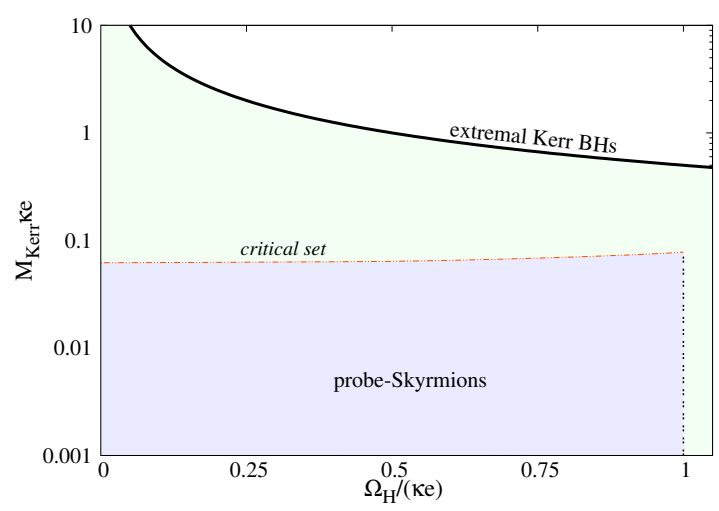

Figure 4. The domain of existence of Skerrmions in a Kerr BH mass vs. horizon angular velocity diagram.
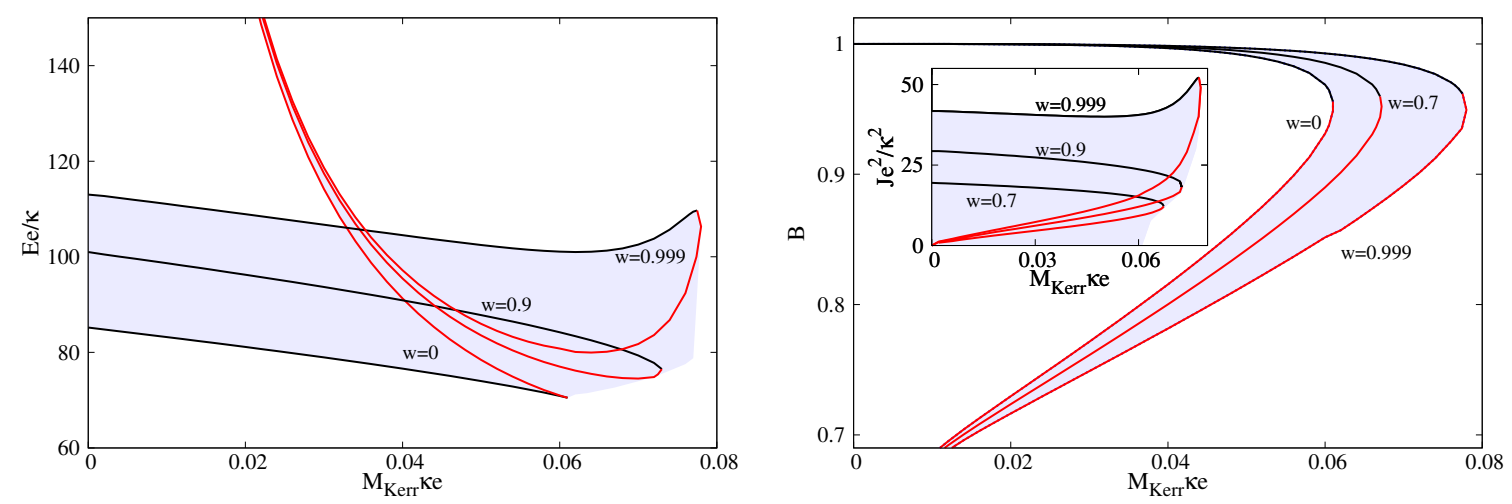

Figure 5. The Skerrmions mass-energy (left panel) or baryon charge (right panel) or angular momentum (inset) ar shown $v s$. the coupling parameter $\kappa e M_{\text {Kerr }}$. The shaded area corresponds to the explored domain of existence.

is observed in the static case, cf. figure 2. In the BH mass vs. horizon angular velocity diagram, the domain of existence of Skerrmions is bounded by a set of Schwarzschild BHs ( $\left.w=\Omega_{H}=0\right)$, the flat spacetime solutions ( $M_{\text {Kerr }}=0$ - not seen in the plot), the maximal frequency line $w=\Omega_{H}=\mu$, and the set of critical backgrounds, wherein the solutions stop to exist, as in the static limit [11].

Thirdly, we have also studied the domain of existence in a Skerrmions mass-energy or angular momentum or baryon number vs. the BH mass (or coupling constant) $M_{\text {Kerr }} \kappa e$ diagram, as shown in figure 5. Figure 5 shows, for a given value of $w$, two branches which exists up to some maximal value of the dimensionless parameter $M_{\text {Kerr }} \kappa e$ (the maximal value increasing with $w$ ). The limiting behaviour on these two branches is similar to the static case $(w=0)$. The solutions on the fundamental branch are connected to flat space spinning Skyrmions, while the limit of the excited branch is approached as $\kappa \rightarrow 0$ and corresponds to the YM model on a fixed Schwarzschild background. In particular, the angular momentum vanishes in this limit, and the above discussion for static solutions is recovered. However, the second $\alpha \rightarrow 0$ limit is difficult to approach numerically. 
Fourthly, the solutions tend to delocalize in the limit $w \rightarrow \mu$, with the mass rapidly increasing, while the numerics becomes challenging.

Finally, the value of the baryon charge $B$ decreases from $B=1$ (the flat space limit) to a minimal nonzero value ${ }^{10}$ which is approached along the second branch as $\alpha \rightarrow 0$. Thus, even in the presence of rotation, the BH cannot "absorbe" the entire Skyrmion, i.e. the Skyrme field does not complete trivialise outside the horizon.

In particular, as seen in figure 4 , we do not find any indication for the occurrence of an existence line.

\section{Spinning BHs with Skyrme hair and non-topological Skerrmions}

We shall now consider the coupled Einstein-Skyrme system, by supplementing (2.1) with the Einstein-Hilbert term. The action of the full model reads

$$
S=\int d^{4} x \sqrt{-g}\left(\frac{R}{16 \pi G}+\mathcal{L}_{S}\right) .
$$

The spinning gravitating solutions are found for the same Skyrme field Ansatz (4.4) and line element (4.1); this time, however, the metric functions $F_{i}$ and $W$ are different from those in the Kerr limit, being found by solving numerically a boundary value problem. Thus, in addition to the Skyrme equations (2.4), we also solve the Einstein equations

$$
G_{\mu \nu}=8 \pi G T_{\mu \nu}
$$

with the stress-energy tensor

$$
\begin{aligned}
T_{\mu \nu}= & -\frac{\kappa^{2}}{2} \operatorname{Tr}\left(K_{\mu} K_{\nu}-\frac{1}{2} g_{\mu \nu} K_{\alpha} K^{\alpha}\right) \\
& -\frac{1}{8 e^{2}} \operatorname{Tr}\left(g^{\alpha \beta}\left[K_{\mu}, K_{\alpha}\right]\left[K_{\nu}, K_{\beta}\right]-\frac{1}{4} g_{\mu \nu}\left[K_{\alpha}, K_{\beta}\right]\left[K^{\alpha}, K^{\beta}\right]\right) \\
& +g_{\mu \nu} m_{\pi}^{2} \operatorname{Tr}\left(\frac{U+U^{\dagger}}{2}-\mathbf{1}\right) .
\end{aligned}
$$

The boundary conditions in the matter sector are similar to those employed in the probe limit, while for the metric functions we impose $\left.F_{i}\right|_{r=\infty}=1,\left.\quad W\right|_{r=\infty}=0,\left.\quad \partial_{\theta} F_{i}\right|_{\theta=0, \pi}=$ $\left.\partial_{\theta} W\right|_{\theta=0, \pi}=0,\left.\quad \partial_{r} F_{i}\right|_{r=r_{H}}=0,\left.\quad W\right|_{r=r_{H}}=\Omega_{H}$.

The ADM mass $M$ and the total angular momentum $J$ of the solutions are read off from the asymptotics of the metric functions,

$$
g_{t t}=-1+\frac{2 G M}{r}+\ldots, \quad g_{\varphi t}=-\frac{2 G J}{r} \sin ^{2} \theta+\ldots,
$$

while the baryon charge $B$ is still given by (4.6).

Following previous studies on hairy BHs [63], it is useful to introduce the parameter

$$
p \equiv \frac{M_{H}}{M}, \text { with } 0 \leqslant p \leqslant 1,
$$

\footnotetext{
${ }^{10}$ Within the numerical errors, this value corresponds to the one found for $w=0$.
} 
where $M_{H}$ is the horizon mass, which measures the BH hairiness: $p=1$ for Kerr BHs, for which $M_{H}=M$ corresponding to no matter fields outside horizon, and $p=0$ for gravitating solitons, for which $M_{H}=0$, since there is no horizon.

Similarly to the test field case, one defines a dimensionless radial coordinate, frequency and a pion mass in terms of the input parameters $\kappa, e$. However, the presence of the Newton's constant $G$ in the theory's action (5.1) introduces a new length scale. Thus, the gravitating model possesses a dimensionless coupling constant

$$
\alpha^{2}=4 \pi G \kappa^{2} .
$$

Consequently, the numerical problem has five input parameters. The first two are geometrical: i) the event horizon angular velocity $\Omega_{H}$; ii) the event horizon radius $r_{H}$ in the metric ansatz (4.1); the next two characterizes the model: iii) the coupling constant $\alpha$ and iv) the (scaled) pion mass $\mu$. The last input parameter ${ }^{11}$ is $\mathbf{v}$ ) the winding number $m$. All displayed numerical results were found for a choice of (scaled) pion mass $\mu=1$ and winding number $m=1$.

\subsection{The topological sector}

The solutions with $B \neq 0$ define the topological sector of spinning BHs with Skyrme hair. The emerging picture in the backreacting case is more complicated than in the probe limit, with new qualitative features, which we have only started to unveil. Its basic features can be summarize as follows.

All known static BHs with Skyrme hair [18] possess rotating generalizations, which are found by increasing gradually the event horizon velocity $\Omega_{H}$. Alternatively, a different route to construct the spinning BH solutions with topological Skyrme hair is to start with the spinning gravitating solitons in [22] (with a given set of relevant parameters) and to slowly increase the horizon size via the parameter $r_{H}$.

Similarly to the test field limit, the synchronous condition (4.5) must hold, with $w=$ $m \Omega_{H}$. Then, the spinning hairy BHs are found for all range $0 \leqslant w<\mu$, where $w, \mu$ are the scaled Skyrme field frequency and mass.

Rotating BHs with Skyrme hair inherit from the solitonic limit [22] a complex branch structure in terms of $\alpha$, a full analysis of which is beyond the scope of this work. Some basic aspects are as follows. For a given value of the (scaled) frequency $w$ and a given horizon size, one set of solutions form the fundamental branch. The limit $\alpha \rightarrow 0$ corresponds to the test field Skerrmions. One finds also a branch of excited solutions ending in the (static) Einstein-YM system as $\alpha \rightarrow 0$ (which corresponds along this branch to $\kappa \rightarrow 0$ ). This is the behaviour found already in the static limit [18]; the arguments therein also applying for $w \neq 0$. However, in the rotating case there exist (at least) two more branches of excited solutions within the full ansatz (4.4), which do not possess a well-defined static limit, nor a Kerr-probe one.

Some basic properties of solutions with a generic value of $\alpha$ as a function of the hairiness parameter $p=M_{H} / M$ can be seen in figure 6 . One can notice e.g. the existence of a

\footnotetext{
${ }^{11}$ As we shall see, the Skyrme field frequency (which is another input parameter) is fixed by $m$ and $\Omega_{H}$ via the synchronization condition (4.5).
} 

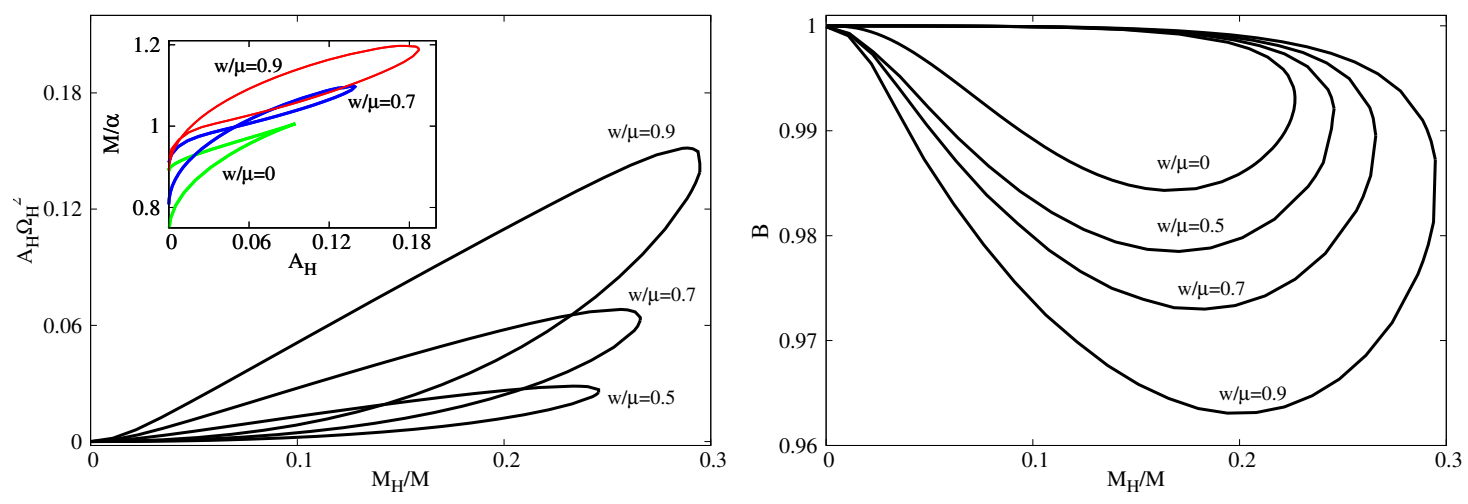

Figure 6. The dimensionless parameter $A_{H} \Omega_{H}^{2}$ (left panel) and the baryon charge $B$ (right panel) as functions of the hairiness parameter $p=M_{H} / M$ for $\mathrm{BH}$ solutions with the coupling constant $\alpha=0.12$.

two branch structure of solutions in terms of $A_{H}$ (or, equivalently the horizon radius $r_{H}$ ), with a maximal value of the horizon area. Again, the properties of the static, spherically symmetric case $(w=0)$ appear to be generic. The lower branch emerges from the (fundamental branch of) gravitating Skyrmions in [22] with a given $\alpha$. There is also a second branch of (excited) solutions which coalesces with the first one for a maximal value of the hairiness parameter $p$. However, in this case the limit $A_{H} \rightarrow 0$ is approached as $\kappa \rightarrow 0$. Then, after a suitable rescaling the fundamental spherically symmetric Bartnik-McKinnon particle-like solution of the Einstein-YM system (as reviewed e.g. in [64]) are recovered.

Most importantly in the context of this work, no indication is found for the presence of an existence line. That is, for all branches, the Skyrme field never trivializes $(p<1)$ and the baryon charge $B$ is always strictly positive, see figure 6 (right panel).

\subsection{The non-topological sector}

The $B \neq 0$ solutions discussed in the previous subsection were found starting with (topologically nontrivial) Skyrme solitons. Disconnected branches of configurations with a vanishing baryon charge $B$ also exist. These solutions are found for a consistent truncation of the Skyrme model with

$$
\pi_{1}+i \pi_{2}=\phi_{1}(r, \theta) e^{i(m \varphi-w t)}, \quad \pi_{3} \equiv 0, \quad \sigma=\phi_{3}(r, \theta), \quad \text { with } \quad \phi_{1}^{2}+\phi_{3}^{2}=1 .
$$

The existence of this Ansatz has been noticed already in [22], the corresponding solitons being dubbed pion clouds. Since $\pi_{3} \equiv 0$, the topological charge vanishes identically in this case, $B=0$. With this truncation, the Skyrme model collapses to the $O(3)$-Fadeev-Skyrme sigma-model [62].

The small field linearization of the model (5.7) around the vacuum state $\sigma=1$ results in the Lagrangean (2.10) with $\chi=0$ and

$$
\psi=\phi_{1}(r, \theta) e^{i(m \varphi-w t)} .
$$

As discussed above, $\psi$ posses bound state solutions for a particular one-dimensional set of Kerr backgrounds forming the existence line. In what follows, we show that these 
'scalar clouds' can be promoted to fully non-linear solutions of the Einstein-Skyrme model, provided one uses the truncated Skyrme Ansatz (5.7).

Following the same approach (and the same boundary conditions) as for the topological sector, we have studied BH solutions with non-topological Skyrme hair, within this particular model. Rather surprisingly, they are akin to the Kerr BHs with scalar hair found in $[25,26]$, sharing most of their features, not to solutions of the full Skyrme model. For example, in strong contrast to the Skyrme case, their existence requires an Einstein term in the action (this holds both for BHs and solitons). As such, they do not possess a flat space limit; moreover, no other solutions on a fixed Kerr background is found apart from the scalar clouds along the existence line.

Figure 7 shows the $\mathrm{BH}$ horizon area for these hairy BHs (normalised as $A_{H} \Omega_{H}^{2}$ ) vs. the hairiness parameter $p=M_{H} / M$. One observes, for instance, that in the limit of a vanishing horizon mass (a $p=0$ hairiness parameter) all solutions reduce to the solitonic 'pion clouds' discussed in [22]. These are a more complicated version of the well-known spinning boson stars [66]. In contrast to the case of solutions of the full model $(B \neq 0)$, one notices the existence of branches of solutions which end on vacuum Kerr BHs $\left(p=M_{H} / M=1\right)$. This feature occurs for a range of field frequencies $0.95<w / \mu<1$. As such those sets of solutions interpolate between solitons and Kerr BHs on the existence line, and they can be considered as nonlinear realizations of the stationary scalar clouds first discussed in [24] within the Einstein-Skyrme system. Branches of BH solutions interpolating between two different solitons with the same field frequency exist as well for small enough values of $w$ (cf. the inset in figure 7). In this case $p$ is always smaller than one.

Finally, the spinning BHs with non-topological Skyrme hair do not possess a static limit, $w=\Omega_{H}=0$. Below a minimal value of the field frequency no hairy BHs are found.

\section{Conclusions}

Static, spherically symmetric BHs with Skyrme hair are a pioneering counter-example to the "no-hair" conjecture, at least in its strongest version. But up to now, the effect of $\mathrm{BH}$ rotation upon this "hair" had not been considered. In this work, we have shown the existence of solutions of the Skyrme model on the Kerr BH background, as test field solutions - Skerrmions - , and also constructed four dimensional spinning BHs with Skyrme hair, which may be regarded as back reacting Skerrmions (see [67] for higher dimensional spinning BHs with Skyrme hair, where a technical advantage occurs).

For the existence of spinning BHs with Skyrme "hair" the synchronisation condition (4.5) is crucial. ${ }^{12}$ We have found there are two sectors of such hairy solutions. The first one is a topological sector qualitatively similar to that found in the static case. The second one is a non-topological sector, qualitatively different from the solutions found in the static case, and more akin to Kerr BHs with synchronised scalar hair [25, 26]. The exploration performed in this work has really just scratched the surface of the rich structure of solutions of the Skyrme-Einstein model, which certainly merits to be analysed in greater depth.

\footnotetext{
${ }^{12}$ The "hair" in the static, spherically symmetric case can be faced as obeying a trivial version of this condition.
} 


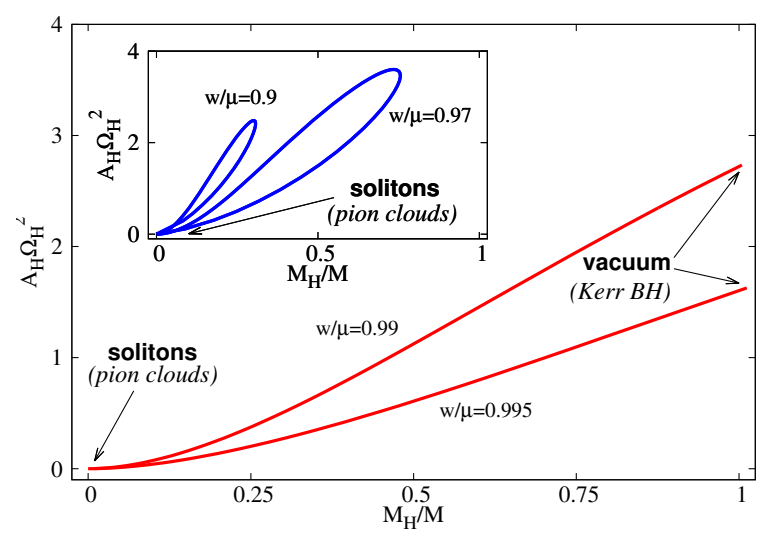

Figure 7. The dimensionless parameter $A_{H} \Omega_{H}^{2}$ as a function of the hairiness parameter $p=M_{H} / M$ for spinning BHs with non-topological Skyrme hair, for a coupling constant $\alpha=10$.

\section{Acknowledgments}

This work has been supported by the FCT (Portugal) IF programme, by the FCT grant PTDC/FISOUT $/ 28407 / 2017$, by the CIDMA (FCT) strategic project UID/MAT/04106/2013, by the CENTRA (FCT) strategic project UID/FIS/00099/2013 and by the European Union's Horizon 2020 research and innovation (RISE) programmes H2020-MSCA-RISE-2015 Grant No. StronGrHEP-690904 and H2020-MSCA-RISE-2017 Grant No. FunFiCO-777740. The authors would like to acknowledge networking support by the COST Action CA16104. Y.S. gratefully acknowledges support from the Ministry of Education and Science of Russian Federation, project No 3.1386.2017. The parallel computations were performed on the cluster HIBRILIT at LIT, JINR, Dubna and Blafis at Aveiro University.

Open Access. This article is distributed under the terms of the Creative Commons Attribution License (CC-BY 4.0), which permits any use, distribution and reproduction in any medium, provided the original author(s) and source are credited.

\section{References}

[1] R. Ruffini and J.A. Wheeler, Introducing the black hole, Phys. Today 24 (1971) 30.

[2] J.D. Bekenstein, Black hole hair: 25 years after, in Physics. Proceedings, 2nd International A.D. Sakharov Conference, Moscow, Russia, 20-24 May 1996, pp. 216-219, gr-qc/9605059 [INSPIRE].

[3] C. Herdeiro and E. Radu, Asymptotically flat black holes with scalar hair: a review, Int. J. Mod. Phys. D 24 (2015) 1542014 [arXiv: 1504.08209] [INSPIRE].

[4] T.P. Sotiriou, Black Holes and Scalar Fields, Class. Quant. Grav. 32 (2015) 214002 [arXiv: 1505.00248] [INSPIRE].

[5] M.S. Volkov, Hairy black holes in the XX-th and XXI-st centuries, in proceedings of 14 th Marcel Grossmann Meeting on Recent Developments in Theoretical and Experimental General Relativity, Astrophysics and Relativistic Field Theories (MG14) (In 4 Volumes), Rome, Italy, 12-18 July 2015, volume 2, pp. 1779-1798, [https://doi.org/10.1142/9789813226609_0184] [arXiv: 1601.08230] [INSPIRE]. 
[6] T.H.R. Skyrme, A Nonlinear field theory, Proc. Roy. Soc. Lond. A 260 (1961) 127 [inSPIRE].

[7] T.H.R. Skyrme, A unified field theory of mesons and baryons, Nucl. Phys. A 31 (1962) 556.

[8] E. Witten, Global Aspects of Current Algebra, Nucl. Phys. B 223 (1983) 422 [InSPIRE].

[9] E. Witten, Current Algebra, Baryons and Quark Confinement, Nucl. Phys. B 223 (1983) 433 [INSPIRE].

[10] C.G. Callan Jr. and E. Witten, Monopole Catalysis of Skyrmion Decay, Nucl. Phys. B 239 (1984) 161 [INSPIRE].

[11] H. Lückock and I. Moss, Black holes have skyrmion hair, Phys. Lett. B 176 (1986) 341 [INSPIRE].

[12] H. Luckock, Black hole skyrmions, in String Theory, Quantum Cosmology and Quantum Gravity, Integrable and Conformal Integrable Theories, H.J. De Vega and N. Sanches eds., World Scientific (1987), p. 455.

[13] S. Droz, M. Heusler and N. Straumann, New black hole solutions with hair, Phys. Lett. B 268 (1991) 371 [INSPIRE].

[14] C. Adam, O. Kichakova, Ya. Shnir and A. Wereszczynski, Hairy black holes in the general Skyrme model, Phys. Rev. D 94 (2016) 024060 [arXiv:1605.07625] [INSPIRE].

[15] G. Dvali and A. Gußmann, Skyrmion Black Hole Hair: Conservation of Baryon Number by Black Holes and Observable Manifestations, Nucl. Phys. B 913 (2016) 1001 [arXiv: 1605.00543] [INSPIRE].

[16] S.B. Gudnason, M. Nitta and N. Sawado, Black hole Skyrmion in a generalized Skyrme model, JHEP 09 (2016) 055 [arXiv: 1605. 07954] [INSPIRE].

[17] N.K. Glendenning, T. Kodama and F.R. Klinkhamer, Skyrme topological soliton coupled to gravity, Phys. Rev. D 38 (1988) 3226 [InSPIRE].

[18] P. Bizon and T. Chmaj, Gravitating skyrmions, Phys. Lett. B 297 (1992) 55 [INSPIRE].

[19] M. Heusler, S. Droz and N. Straumann, Stability analysis of selfgravitating skyrmions, Phys. Lett. B 271 (1991) 61 [INSPIRE].

[20] M. Heusler, N. Straumann and Z.-h. Zhou, Selfgravitating solutions of the Skyrme model and their stability, Helv. Phys. Acta 66 (1993) 614 [inSPIRE].

[21] R.A. Battye, S. Krusch and P.M. Sutcliffe, Spinning skyrmions and the skyrme parameters, Phys. Lett. B 626 (2005) 120 [hep-th/0507279] [INSPIRE].

[22] T. Ioannidou, B. Kleihaus and J. Kunz, Spinning gravitating skyrmions, Phys. Lett. B 643 (2006) 213 [gr-qc/0608110] [INSPIRE].

[23] I. Perapechka and Ya. Shnir, Spinning gravitating Skyrmions in a generalized Einstein-Skyrme model, Phys. Rev. D 96 (2017) 125006 [arXiv:1710.06334] [inSPIRE].

[24] S. Hod, Stationary Scalar Clouds Around Rotating Black Holes, Phys. Rev. D 86 (2012) 104026 [Erratum ibid. D 86 (2012) 129902] [arXiv:1211.3202] [InSPIRE].

[25] C. Herdeiro and E. Radu, Kerr black holes with scalar hair, Phys. Rev. Lett. 112 (2014) 221101 [arXiv: 1403.2757 ] [INSPIRE].

[26] C. Herdeiro and E. Radu, Construction and physical properties of Kerr black holes with scalar hair, Class. Quant. Grav. 32 (2015) 144001 [arXiv:1501.04319] [INSPIRE].

[27] Y. Brihaye, C. Herdeiro and E. Radu, Myers-Perry black holes with scalar hair and a mass gap, Phys. Lett. B 739 (2014) 1 [arXiv:1408.5581] [InSPIRE].

[28] B. Kleihaus, J. Kunz and S. Yazadjiev, Scalarized Hairy Black Holes, Phys. Lett. B 744 (2015) 406 [arXiv: 1503.01672] [INSPIRE]. 
[29] C. Herdeiro, J. Kunz, E. Radu and B. Subagyo, Myers-Perry black holes with scalar hair and a mass gap: Unequal spins, Phys. Lett. B 748 (2015) 30 [arXiv:1505.02407] [INSPIRE].

[30] C. Herdeiro, E. Radu and H. Rúnarsson, Kerr black holes with self-interacting scalar hair: hairier but not heavier, Phys. Rev. D 92 (2015) 084059 [arXiv: 1509. 02923] [INSPIRE].

[31] C. Herdeiro, E. Radu and H. Runarsson, Kerr black holes with Proca hair, Class. Quant. Grav. 33 (2016) 154001 [arXiv: 1603.02687] [INSPIRE].

[32] J.F.M. Delgado, C. Herdeiro, E. Radu and H. Runarsson, Kerr-Newman black holes with scalar hair, Phys. Lett. B 761 (2016) 234 [arXiv:1608.00631] [INSPIRE].

[33] C. Herdeiro and E. Radu, Spinning boson stars and hairy black holes with nonminimal coupling, Int. J. Mod. Phys. D 27 (2018) 1843009 [arXiv:1803.08149] [InSPIRE].

[34] C. Herdeiro, J. Kunz, E. Radu and B. Subagyo, Probing the universality of synchronised hair around rotating black holes with Q-clouds, Phys. Lett. B 779 (2018) 151 [arXiv:1712.04286] [INSPIRE].

[35] C. Herdeiro, E. Radu and H. Runarsson, Non-linear Q-clouds around Kerr black holes, Phys. Lett. B 739 (2014) 302 [arXiv:1409.2877] [INSPIRE].

[36] R. Emparan and H.S. Reall, A Rotating black ring solution in five-dimensions, Phys. Rev. Lett. 88 (2002) 101101 [hep-th/0110260] [INSPIRE].

[37] S.R. Coleman, Q Balls, Nucl. Phys. B 262 (1985) 263 [Erratum ibid. B 269 (1986) 744] [INSPIRE].

[38] M.S. Volkov and E. Wohnert, Spinning Q balls, Phys. Rev. D 66 (2002) 085003 [hep-th/0205157] [INSPIRE].

[39] B. Kleihaus, J. Kunz and M. List, Rotating boson stars and Q-balls, Phys. Rev. D 72 (2005) 064002 [gr-qc/0505143] [INSPIRE].

[40] S. Hod, Kerr-Newman black holes with stationary charged scalar clouds, Phys. Rev. D 90 (2014) 024051 [arXiv: 1406.1179] [INSPIRE].

[41] C.L. Benone, L.C.B. Crispino, C. Herdeiro and E. Radu, Kerr-Newman scalar clouds, Phys. Rev. D 90 (2014) 104024 [arXiv:1409.1593] [INSPIRE].

[42] J. Wilson-Gerow and A. Ritz, Black hole energy extraction via a stationary scalar analog of the Blandford-Znajek mechanism, Phys. Rev. D 93 (2016) 044043 [arXiv:1509.06681] [INSPIRE].

[43] C. Bernard, Stationary charged scalar clouds around black holes in string theory, Phys. Rev. D 94 (2016) 085007 [arXiv: 1608.05974] [INSPIRE].

[44] I. Sakalli and G. Tokgoz, Stationary Scalar Clouds Around Maximally Rotating Linear Dilaton Black Holes, Class. Quant. Grav. 34 (2017) 125007 [arXiv:1610.09329] [INSPIRE].

[45] S. Hod, Spinning Kerr black holes with stationary massive scalar clouds: The large-coupling regime, JHEP 01 (2017) 030 [arXiv:1612.00014] [INSPIRE].

[46] H.R.C. Ferreira and C. Herdeiro, Stationary scalar clouds around a BTZ black hole, Phys. Lett. B 773 (2017) 129 [arXiv:1707.08133] [INSPIRE].

[47] G.H. Derrick, Comments on nonlinear wave equations as models for elementary particles, J. Math. Phys. 5 (1964) 1252 [inSPIRE].

[48] C. Adam, J. Sanchez-Guillen and A. Wereszczynski, A Skyrme-type proposal for baryonic matter, Phys. Lett. B 691 (2010) 105 [arXiv:1001.4544] [INSPIRE].

[49] C. Adam, J. Sanchez-Guillen and A. Wereszczynski, A BPS Skyrme model and baryons at large $N_{c}$, Phys. Rev. D 82 (2010) 085015 [arXiv:1007.1567] [INSPIRE]. 
[50] I. Perapechka and Ya. Shnir, Crystal structures in generalized Skyrme model, Phys. Rev. D 96 (2017) 045013 [arXiv: 1703.10673] [INSPIRE].

[51] M.J. Esteban, A Direct Variational Approach to Skyrme's Model for Meson Fields, Commun. Math. Phys. 105 (1986) 571 [INSPIRE].

[52] N.S. Manton and P. Sutcliffe, Topological solitons, Cambridge University Press (2004).

[53] S. Krusch and P. Sutcliffe, Sphalerons in the Skyrme model, J. Phys. A 37 (2004) 9037 [hep-th/0407002] [INSPIRE].

[54] Ya. Shnir and D.H. Tchrakian, Skyrmion-Anti-Skyrmion Chains, J. Phys. A 43 (2010) 025401 [arXiv: 0906 . 5583] [INSPIRE].

[55] I. Smolić, Symmetry inheritance of scalar fields, Class. Quant. Grav. 32 (2015) 145010 [arXiv: 1501.04967] [INSPIRE].

[56] H. Boutaleb-Joutei, A. Chakrabarti and A. Comtet, Gauge Field Configurations in Curved Space-times, Phys. Rev. D 20 (1979) 1884 [INSPIRE].

[57] S.B. Gudnason and M. Nitta, Higher-order Skyrme hair of black holes, JHEP 05 (2018) 071 [arXiv: 1803.10786] [INSPIRE].

[58] O. Schenk and K. Gärtner Solving unsymmetric sparse systems of linear equations with PARDISO, Future Gener. Comp. Sy. 20(3) (2004) 475.

[59] W. Schönauer and R. Weiß, Efficient vectorizable PDE solvers, J. Comput. Appl. Math. 27 (1989) 279.

[60] M. Schauder, R. Weiß and W. Schönauer, The CADSOL Program Package, Universität Karlsruhe, Interner Bericht Nr. 46/92 (1992).

[61] R. Rajaraman, Solitons and Instantons: An Introduction to Solitons and Instantons in Quantum Field Theory, North-Holland Publishing Company (1982).

[62] E. Radu and M.S. Volkov, Existence of stationary, non-radiating ring solitons in field theory: knots and vortons, Phys. Rept. 468 (2008) 101 [arXiv:0804.1357] [InSPIRE].

[63] J.F.M. Delgado, C. Herdeiro and E. Radu, Violations of the Kerr and Reissner-Nordström bounds: Horizon versus asymptotic quantities, Phys. Rev. D 94 (2016) 024006 [arXiv: 1606.07900] [INSPIRE].

[64] M.S. Volkov and D.V. Gal'tsov, Gravitating nonAbelian solitons and black holes with Yang-Mills fields, Phys. Rept. 319 (1999) 1 [hep-th/9810070] [INSPIRE].

[65] S.R. Brandt and E. Seidel, The Evolution of distorted rotating black holes. 3: Initial data, Phys. Rev. D 54 (1996) 1403 [gr-qc/9601010] [InSPIRE].

[66] S. Yoshida and Y. Eriguchi, Rotating boson stars in general relativity, Phys. Rev. D 56 (1997) 762 [INSPIRE].

[67] Y. Brihaye, C. Herdeiro, E. Radu and D.H. Tchrakian, Skyrmions, Skyrme stars and black holes with Skyrme hair in five spacetime dimension, JHEP 11 (2017) 037 [arXiv:1710.03833] [INSPIRE]. 